\title{
Certification of Antibiotics
}

\author{
By HENRY WELCH, Ph.D.
}

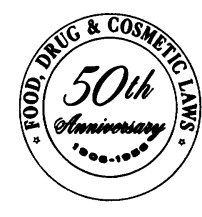

It is generally agreed that little if any good is accomplished by wars. Penicillin perhaps is the exception, for its development, rapid availability, and wide use stemmed from the great need for the drug in World War II. Similarly, the antibiotics certification program got its initial impetus because of our entrance into this worldwide conflict.

In 1941 there was insufficient penicillin in the United States to treat a single case, and in 1942 probably not enough to treat a hundred cases. However, by September 1943 there was sufficient penicillin to satisfy the early demands of the Armed Forces of this country and those of our allies.

It was during September 1943, at the request of the Armed Forces and the War Production Board of the United States, that the Food and Drug Administration undertook the testing of each lot of penicillin produced, for potency, sterility, toxicity, pyrogenicity, $\mathrm{pH}$, and moisture content. On the basis of the results obtained by these tests, the lot was either accepted or rejected.

By June 1945 there was sufficient penicillin to satisfy not only the Armed Forces but a great civilian demand as well, and there was an ap-

Dr. Welch is director of the Division of Antibiotics, Food and Drug Administration. Since its beginning in 1943, he has been in charge of the FDA's antibiotics certification program. He is a co-author of the books, Antibiotic Therapy (1951) and Principles and Practices of Antibiotic Therapy (1954); the editor of the Manual of Antibiotics (1954); and the editor in chief of the journals, "Antibiotics and Chemotherapy" and "Antibiotic Medicine." parent need for a method of control for this important therapeutic agent. On July 6, 1945, the Federal Food, Drug, and Cosmetic Act was amended to require the certification of batches of drugs composed wholly or partly of any kind of penicillin. The Federal Security Administrator, in accordance with the provisions of this amendment, promulgated regulations providing such standards of identity, strength, quality, and purity as would insure the safety and effcacy of penicillin and penicillin preparations.

Under the law, each manufacturer, before distributing a batch of penicillin in interstate commerce, must submit samples of the batch to the Food and Drug Administration for examination and must obtain a certificate showing that it complies with applicable regulations. Each product proposed for certification to the Food and Drug Administration must be shown to be safe and efficacious, this proof being based on actual clinical trial.

In 1947 the Federal Food, Drug, and Cosmetic Act was amended to include streptomycin and its derivatives. In 1949 it was further amended to include aureomycin (chlortetracycline), chloramphenicol (chloromycetin), and bacitracin. Tetracycline, being a derivative of aureomycin, is also certified by the Government.

There have been no amendments to the act since 1949 to require the certification of antibiotics developed since that time. Therefore, all other currently available antibiotics are subject only to the general provisions of the act, unless they are added to one of the antibiotics for which certification is required.

\section{Growth of the Antibiotics Industry}

When penicillin was first produced in the United States, the potency and hence the purity 
was quite low. Early assays of this drug indicated average potency ranges from 100 to 200 units per milligram. The purity of penicillin improved rapidly in the first few years of manufacture, and the crude material, consisting of a dark brown, amorphous powder, gradually changed in color and consistency to a light brown, then yellow, and finally, the white crystalline form. Production amounted to only about 29 pounds in 1943.

When penicillin became available for the civilian population in 1945 , the purity of the drug had been improved considerably so that the calcium salt, which was the one used to the greatest extent, had a potency on the order of 1,200 units per milligram. The crystalline drug (sodium penicillin), which soon appeared, had a potency of 1,667 units per milligram.

During the war years and for some time thereafter, 20 manufacturers in this country produced practically the entire world's supply of penicillin. Competition as such did not exist. All that was produced was consumed and the price was high. The Army paid as much as $\$ 20$ for each 100,000 units. By contrast, in late 1955 the Army purchased penicillin in vials packed in wooden cases for shipment overseas for 5 cents for each 300,000 units; that is, less than 2 cents per 100,000 units. Competition now is extremely keen, and instead of 20 manufacturers of penicillin, there are only 9 . In fact, 4 of these 9 account for some 70 percent of the production. In addition to the 9 manufacturers of penicillin, there are 4 others producing antibiotics. These 13 manufacturers are responsible for the great bulk of the world's supply of these important drugs.

Production of penicillin in this country started an industry that today has a capital worth of about $\$ 1$ billion. In less than 15 years a number of other antibiotic drugs have found their way into the therapy of infectious diseases. In table 1 are the 24 antibiotics that have been shown to be clinically useful of the 3,000 to 4,000 that have been described in the literature. In addition to these 24, hundreds of others are awaiting tests on the shelves of those manufacturers still in the antibiotics field.

Changes in production and approximate dollar value of the seven antibiotics that are
Table 1. Clinically useful antibiotics

\begin{tabular}{|c|c|}
\hline Antibiotic & $\begin{array}{c}\text { Year } \\
\text { developed }\end{array}$ \\
\hline 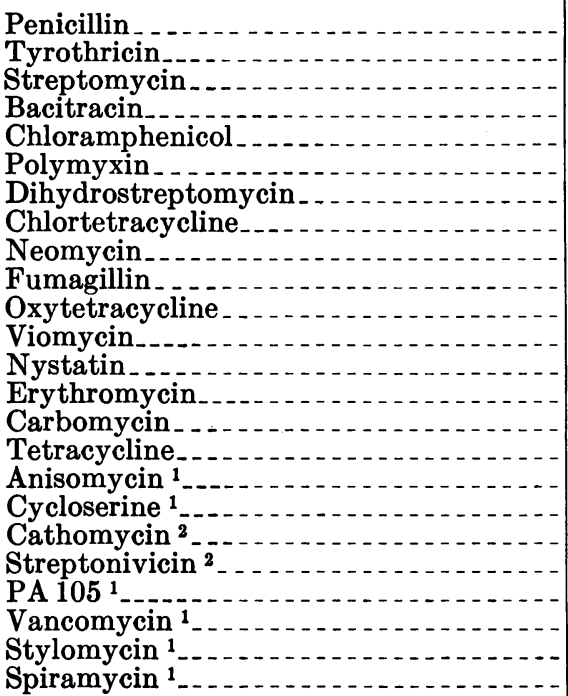 & $\begin{array}{c}1929-1943 \\
1939 \\
1943 \\
1945 \\
1947 \\
1947 \\
1948 \\
1948 \\
1949 \\
1950 \\
1950 \\
1950 \\
1951 \\
1952 \\
1952 \\
1953 \\
1954 \\
1955 \\
1955 \\
1955 \\
1955 \\
1955 \\
1955 \\
1955\end{array}$ \\
\hline
\end{tabular}

1 Still under clinical study.

2 Although isolated from different sources, cathomycin and streptonivicin have been shown to be the same antibiotic.

used to the greatest extent-penicillin, streptomycin, dihydrostreptomycin, and the broadspectrum antibiotics-are shown in table 2 . Only 29 pounds of crude penicillin were produced in 1943, and this amount had a value at the manufacturers' level of about $\$ 3$ million. Production increased more than 100 times in 1944. In 1945 the original estimate of 5,000 kg., which was expected to be sufficient to supply the world demand, was exceeded by a considerable amount: Fourteen thousand pounds, or more than 6,000 kg., were produced. Competition in the industry became extremely keen from 1951 on, and although 756,000 pounds were produced in 1953, the dollar value was less than half that of the 636,000 pounds produced in 1951.

The history of the production of streptomycin is not markedly different. The experience in production methods obtained with penicillin resulted in some 3,800 pounds being produced in 1946, the first year that streptomycin was available commercially. This drug, too, when first used was expensive, in the neighborhood of $\$ 15$ per gram. The competition in penicillin was re- 
flected in the price of streptomycin by 1953, and although 375,000 pounds were produced, 100 times as much as in 1946, the value increased only 3 times.

More than 417,000 pounds of the broad-spectrum drugs were produced in 1953, with an approximate value of $\$ 137$ million. In 1954 , 440,000 pounds valued at $\$ 150$ million were made available.

The broad-spectrum antibiotics and penicillin have uses other than in the prophylaxis and treatment of disease. They are used, for example, in the promotion of animal growth, and in 1954 some 490,000 pounds were produced for feed supplements. The total for all antibiotics in 1954 was some 2,284,000 pounds, with an approximate value at the manufacturers' level of more than $\$ 272$ million. According to recent estimates, production was not materially different in 1955.

A large number of antibiotic preparations are now available to the physician in this country. Among those preparations sold in greatest quantity, there are more than 300 available for clinical use. Preparations containing penicillin number 150 . There are 38 streptomycin and dihydrostreptomycin preparations, 47 chlortetracycline and tetracycline preparations, 25 oxy-

\section{Table 2. Antibiotic production in the United} States, 1943-55

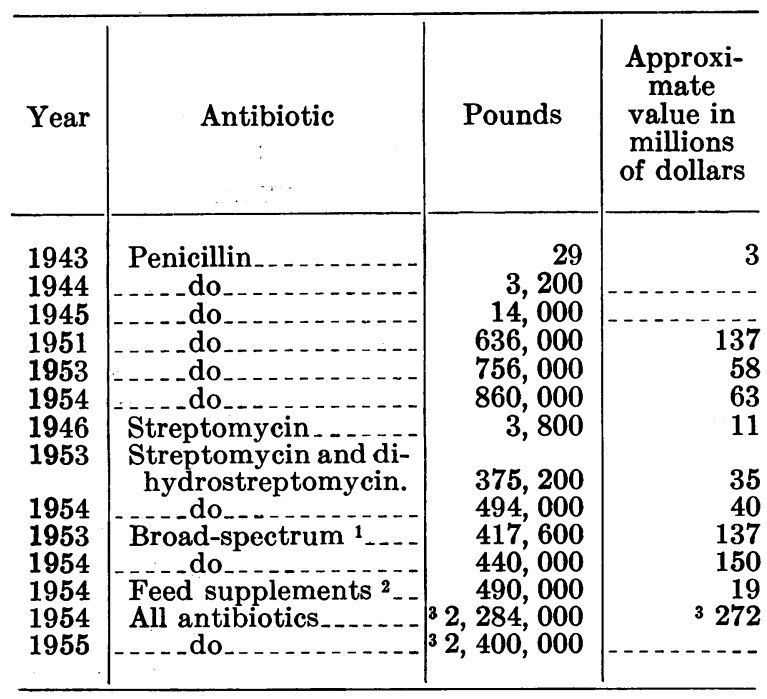

1 Chlortetracycline, oxy tetracycline, tetracycline, and chloramphenicol.

2 The broad-spectrum antibiotics and penicillin.

8 Estimated. tetracycline preparations, 15 chloramphenicol preparations, and 27 bacitracin preparations.

\section{Certification Practices and Principles}

At the start of penicillin control in 1943, a group of six technicians of the Food and Drug Administration, in collaboration with other governmental agencies, successfully checked for the Armed Forces all penicillin then produced. The apparatus used was hand built and makeshift to meet the requirements of a group of new methods.

As time went on and new antibiotics came into clinical use, the laboratory force was expanded. New and adequate equipment became available, and by 1950 the staff controlling certifiable antibiotics numbered 80 . Instead of milligrams of penicillin worth more than its weight in gold, lots containing hundreds of thousands of vials of penicillin were being assayed, and the glass used for packaging cost more than the drug. Assays were performed on a mass scale with automatic equipment (fig. 1), and special reading devices sped up potency determinations and made them more accurate (fig. 2). Electronic equipment for infrared analysis (fig. 3) and for accurate, automatic temperature determinations (fig. 4) simplified considerably the problems of mass assays.

Since its beginning in 1945 , the certification program has been self-sustaining. All equipment is purchased from fees paid by the producers of the certifiable antibiotics. In addition, since it is set up on a cost basis, excess fees are returned quarterly to the manufacturers on a pro rata basis.

Under the certification system a producer of a new antibiotic preparation for which certification is required presents to the Food and Drug Administration clinical data demonstrating the safety and efficacy of his proposed product. If the data are found to be satisfactory, a monograph is prepared establishing standards of identity, strength, quality, and purity. Thereafter, samples of each batch produced are forwarded to the Division of Antibiotics for examination, and a certificate is issued on all batches complying with the standards. At the present time some 20,000 batches of antibiotics and their preparations are examined and certified yearly. 
Antibiotic preparations destined for export are not required to be certified under the certification system. Such preparations are exempt under section 801 (d) of the Federal Food, Drug, and Cosmetic Act, provided they meet the specifications of the foreign buyer, are not in violation of the laws of the country to which they are to be shipped, and are marked clearly for export. The buyer of the foreign country must require that the certifiable drugs pur-

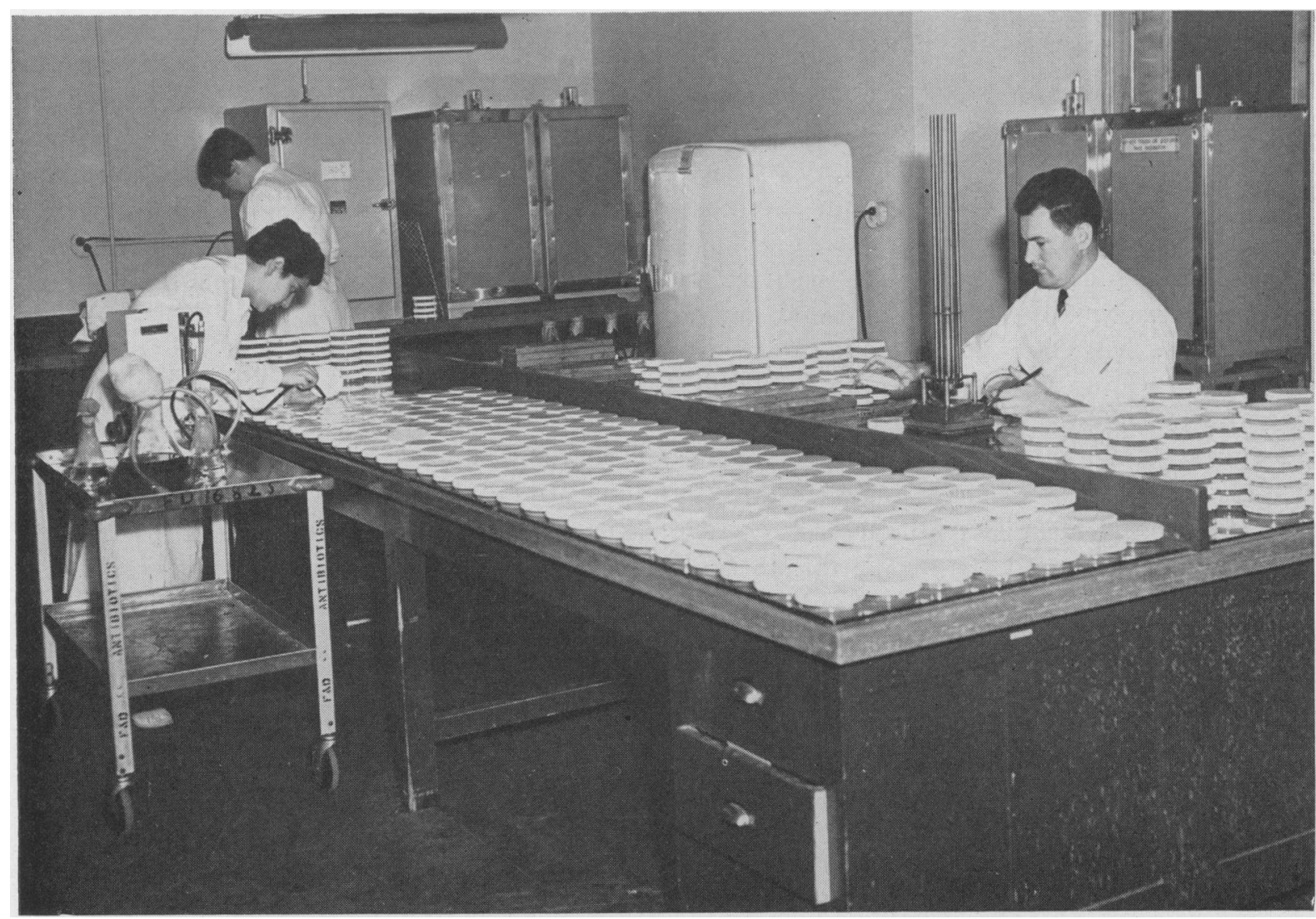

Figure 1. Preparing agar plates for penicillin assays.

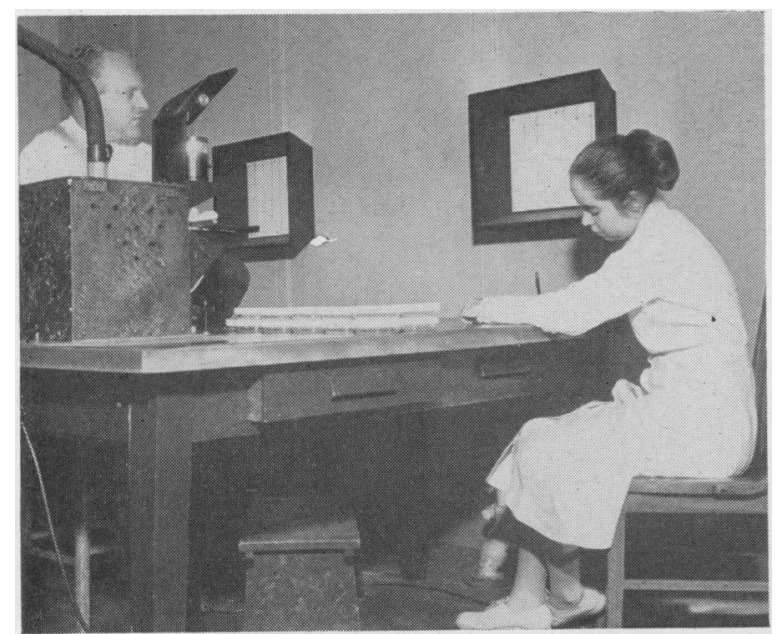

Figure 2. Measuring zones of inhibition to determine potency of antibiotics.

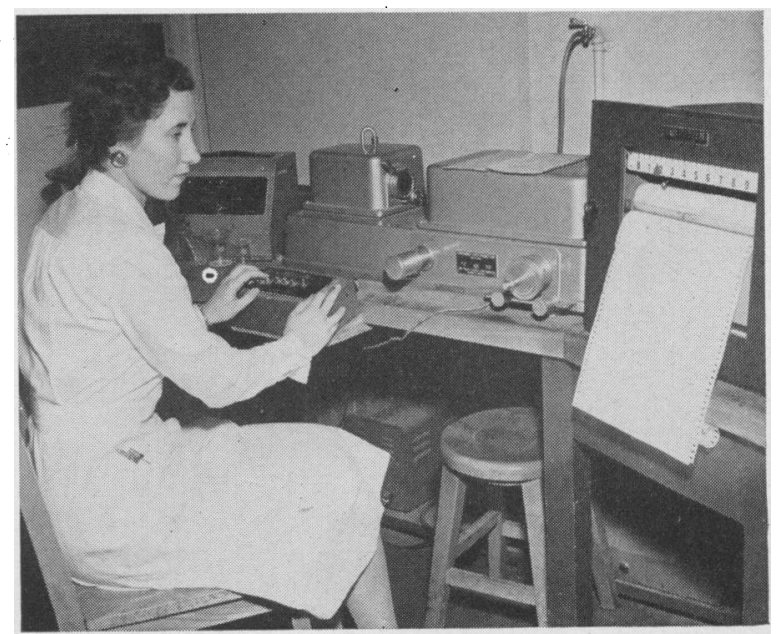

Figure 3. Electronic equipment used for infrared analysis of antibiotics. 
chased meet United States Government standards if he wishes to obtain certified material. If he does this, the preparations for export must be sampled, tested, and certified before shipment. Consequently, some of the United States producers certify all of their preparations whether destined for the domestic or export market.

Since July 1945, nearly 300 companies have used the certification services. During this period of approximately 10 years, 150,000 batches of various preparations of penicillin, streptomycin, dihydrostreptomycin, chlortetracycline, tetracycline, chloramphenicol, and bacitracin have been examined. Taking the usual daily dose of these drugs to be 300,000 units for penicillin, 60,000 units for bacitracin, and 1 gram each for streptomycin, dihydrostreptomycin, chlortetracycline, chloramphenicol, and tetracycline, the quantity of these drugs examined represents approximately 7 billion daily doses. The average cost paid by the producers for certification of each daily dose was 7/100 of a cent. Over 1,000 batches have been either rejected by FDA or withdrawn by the producer because they were substandard. These substandard batches which were prevented from reaching the consumer represent more than 70 million daily doses.

During the past 5 years, the Administrator of the Federal Security Agency, and later the Secretary of the Department of Health, Education, and Welfare, has found that certification of the antibiotic preparations in the list on page 599 is not necessary to insure safety and efficacy. These preparations are now exempt from certification.

\section{The Impact of Antibiotics}

Through use of antibiotics the spectrum of amenable diseases has widened almost yearly. Either completely or partially controlled by the seven antibiotics previously mentioned are

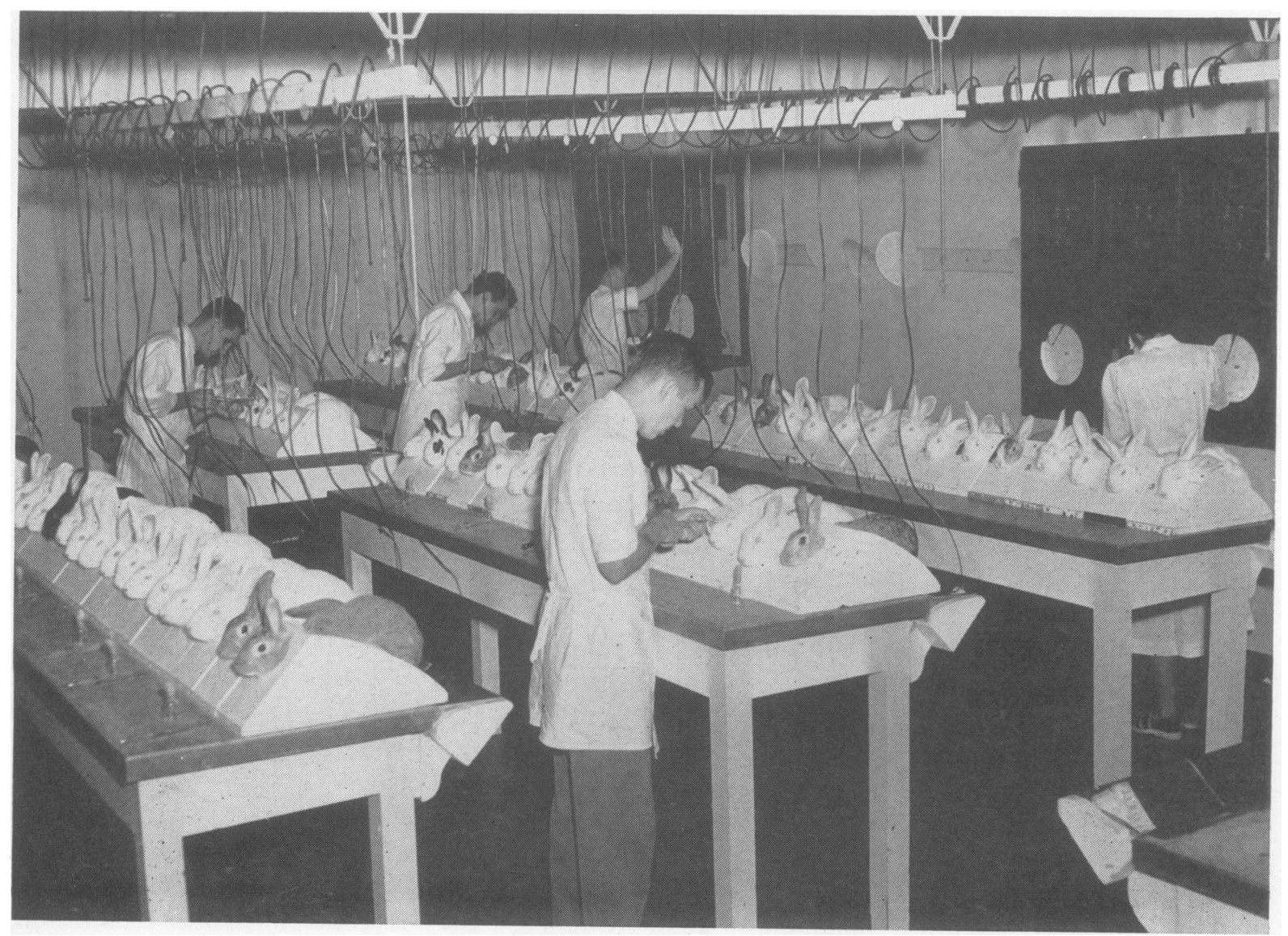

Figure 4. Electronic equipment for automatically determining rectal temperatures of rabbits. 


\section{Antibiotic Preparations Now Exempt From Certification}

Crystalline penicillin $\mathrm{G}$ sodium.

Crystalline penicillin $G$ potassium.

Antibiotics for diagnostic use.

Antibiotics for fish diseases.

Antibiotics as preservatives for bull semen.

Antibiotics as preservatives for biological drugs.

Antibiotics for use as ingredients of animal feed for certain prescribed conditions.

Animal feed mixes intended for certain prescribed conditions.

Buffered crystalline penicillin G sodium.

Buffered crystalline penicillin $G$ potassium.

Certain formulations of penicillin troches.

Certain formulations of bacitracin ointment.

Streptomycin sulfate granules (powder), oral veterinary.

Crude chlortetracycline, oral veterinary.
Antibiotics for agricultural use.

Buffered penicillin powder, oral veterinary.

Penicillin-streptomycin (penicillin-dihydrostreptomycin) tablets, oral veterinary.

Penicillin-streptomycin (penicillin-dihydrostreptomycin) powder, oral veterinary.

Streptomycin (dihydrostreptomycin) for inhalation therapy, veterinary.

Streptomycin hydrochloride (sulfate) solution, oral veterinary.

Chlortetracycline (chlortetracycline hydrochloride) powder, veterinary; tetracycline (tetracycline hy. drochloride) powder, veterinary.

Soluble bacitracin methylene disalicylate, oral veterinary.

Bacitracin powder, oral veterinary. pneumonococcal and streptococcal infections, meningococcal and gonococcal infections, staphylococcal infections, syphilis, yaws, pinta, bejel, Weil's disease, relapsing fever, tropical ulcer, Vincent's angina, and many surgical, urinary, and intestinal tract infections; amebiasis, brucellosis, plague, tularemia, and tuberculosis; and diseases due to certain large viruses and rickettsiae, including primary atypical pneumonia, psittacosis, lymphogranuloma venereum, trachoma, Rocky Mountain spotted fever, epidemic and endemic typhus, scrub typhus, and $\mathrm{Q}$ fever. Also largely under control are granuloma inguinale, chancroid, Carrion's disease, pertussis, diphtheria, influenzal meningitis, anthrax, actinomycosis, trichomonas vaginalis vaginitis, and rat bite fever. In addition to use against these specific infections, these antibiotics have found wide use in ophthalmology and in oral surgery and dentistry.

This is indeed an imposing list. However, a second line of defense against a variety of infections is also available. Tyrothricin, bacitracin, polymyxin, neomycin, viomycin, erythromycin, carbomycin, and fumagillin all have a place in the physician's armamentarium. These often prove to be successful where the others fail. Furthermore, antibiotics just becoming available may add new ammunition in the war against infectious diseases.
I think it may be safely said that the antibiotic preparations are used as prescription drugs more frequently in treating serious and crippling diseases than any other drug or class of drugs now available. It has been conservatively estimated that in dollars spent for prescription drugs in recent years, 40 percent of all prescriptions drugs sold include one or more antibiotics.

Thus it is obvious that these drugs are of the greatest importance to the public health and that they deserve a special form of control, such as that established by the certification system. The principle on which certification of these drugs is based appears to be sound; that is, they must be certified because of $(a)$ their great importance to the public health, $(b)$ their use in serious and crippling diseases, $(c)$ the security of a double check on their safety and efficacy given the practicing physician and his patients, and $(d)$ their production by biological means, which may result in vagaries in manufacture and assay. Furthermore, the manufacturer is protected adequately from restrictive or paternalistic control by the principle that an antibiotic shall be exempt from certification when the Secretary of the Department of Health, Education, and Welfare feels that it is no longer in the interest of the public health to continue certification. 